\title{
Association of caspase-1 polymorphisms with Chagas cardiomyopathy among individuals in Santa Cruz, Bolivia
}

\author{
Katherine Yih-Jia Fu ${ }^{[1]}$, Roxana Zamudio ${ }^{[2]}$, Jo Henderson-Frost ${ }^{[3]}$, Alex Almuedo ${ }^{[4]}$, \\ Hannah Steinberg ${ }^{[5]}$, Steven Joseph Clipman ${ }^{[5]}$, Gustavo Duran ${ }^{[6]}$, \\ Rachel Marcus ${ }^{[7]}$, Thomas Crawford ${ }^{[8]}$, Daniel Alyesh ${ }^{[8]}$, Rony Colanzi ${ }^{[9]}$, \\ Jorge Flores ${ }^{[6]}$, Robert Hugh Gilman ${ }^{[5]}$ and Caryn Bern ${ }^{[10]}$
}

\begin{abstract}
[1]. Rutgers Robert Wood Johnson Medical School, New Brunswick, New Jersey, United States of America. [2]. Department of Genetics, University of Leicester, Leicester, United Kingdom. [3]. Massachusetts General Hospital, Boston, Massachusetts, United States of America. [4]. Fundació Hospital de Granollers, Granollers, Barcelona, Spain. [5]. Department of International Health, Johns Hopkins Bloomberg School of Public Health, Baltimore, Maryland, United States of America. [6]. Hospital San Juan de Dios, Santa Cruz de la Sierra, Bolivia. [7]. MedStar Washington Hospital Center, Washington, District of Columbia, United States of America. [8]. Division of Cardiology, University of Michigan, Ann Arbor, Michigan, United States of America. [9]. Hospital Japones, Santa Cruz de la Sierra, Bolivia. [10]. Department of Epidemiology and Biostatistics, University of California San Francisco School of Medicine, San Francisco, California, United States of America.
\end{abstract}

\begin{abstract}
Introduction: Trypanosoma cruzi $(T c)$ infection is usually acquired in childhood in endemic areas, leading to Chagas disease, which progresses to Chagas cardiomyopathy in $20-30 \%$ of infected individuals over decades. The pathogenesis of Chagas cardiomyopathy involves the host inflammatory response to $T$. cruzi, in which upstream caspase- 1 activation prompts the cascade of inflammatory chemokines/cytokines, cardiac remodeling, and myocardial dysfunction. The aim of the present study was to examine the association of two caspase-1 single nucleotide polymorphisms (SNPs) with cardiomyopathy. Methods: We recruited infected $\left(T c^{+}, \mathrm{n}=149\right)$ and uninfected $\left(T c^{-}, \mathrm{n}=87\right)$ participants in a hospital in Santa Cruz, Bolivia. Cardiac status was classified (I, II, III, IV) based on Chagas cardiomyopathy-associated electrocardiogram findings and ejection fractions on echocardiogram. Genotypes were determined using Taqman probes via reverse transcription-polymerase chain reaction of peripheral blood DNA. Genotype frequencies were analyzed according to three inheritance patterns (dominant, recessive, additive) using logistic regression adjusted for age and sex. Results: The AA allele for the caspase-1 SNP rs501192 was more frequent in $T_{\mathcal{C}}+$ cardiomyopathy (classes II, III, IV) patients compared to those with a normal cardiac status (class I) [odds ratio $(\mathrm{OR})=-2.18, \mathrm{p}=0.117]$. This trend approached statistical significant considering only $T_{c}+$ patients in class I and II $(\mathrm{OR}=-2.64$, $\mathrm{p}=0.064$ ). Conclusions: Caspase-1 polymorphisms may play a role in Chagas cardiomyopathy development and could serve as markers to identify individuals at higher risk for priority treatment.
\end{abstract}

Keywords: Chagas cardiomyopathy. .Single nucleotide polymorphisms. Caspase-1.

\section{INTRODUCTION}

Chagas disease is the most important parasitic disease in the Western hemisphere, responsible for a disease burden that is estimated to be 7 times higher than that of malaria in this region ${ }^{1}$. Chagas disease is caused by Trypanosoma cruzi infection, which is most commonly acquired in childhood in endemic areas but often remains clinically silent, and therefore undiagnosed, for decades. Vector-borne transmission is focused on endemic rural communities with poor housing conditions. However, migration has brought people living with Chagas disease to several cities in Latin America, North America, and Europe ${ }^{2,3}$.

Corresponding author: Dr. Katherine Fu.

e-mail: kayfu01@gmail.com

Received 9 February 2017

Accepted 16 August 2017
Although the majority of infected individuals will never develop overt symptomatic disease, $20-30 \%$ of cases will progress to Chagas cardiomyopathy, characterized by conduction system deficits, brady- and tachyarrhythmias, eventually leading to progressive dilated cardiomyopathy ${ }^{4}$. In one large public hospital in Santa Cruz, Bolivia, $60 \%$ of the cases of congestive heart failure and $79 \%$ of cases of advanced heart failure were associated with $T$. cruzi infection ${ }^{5}$. Once dilated cardiomyopathy is present, the patients' short-term mortality rates are high ${ }^{6}$. Anti-trypanosomal therapy in the early chronic phase is associated with a cure rate of $60 \%$ based on serology, and this treatment is presumed to decrease or eliminate the likelihood of future cardiac progression ${ }^{7,8}$. However, once structural heart disease is present, anti-trypanosomal therapy does not appear to influence disease progression?. Therefore, identifying early indicators of Chagas cardiomyopathy risk would help to best focus treatment on those with the highest probability of future morbidity. 
The pathogenesis of Chagas cardiomyopathy includes a strong immunological component ${ }^{10}$. Control of the parasite soon after infection requires a robust inflammatory immune response. The acute phase ends when T. cruzi replication is suppressed by an effective T helper cell 1 response. Nevertheless, the infection persists in the absence of treatment, and failure to appropriately down-regulate the inflammatory response appears to play a central role in the pathogenesis of chronic Chagas cardiomyopathy ${ }^{11,12}$. The innate immune response against T. cruzi involves recruitment of the NLRP3 (nod-like receptor family pyrin domain containing 3 ) inflammasome with a caspase recruitment domain ${ }^{13}$. Inflammasomes are cytosolic receptors that respond in the face of infection to control morbidity; however, their excessive activation can also contribute to the ongoing pathology ${ }^{14}$. Upon NLRP3 inflammasome recruitment, pro-caspase-1 is cleaved, thereby activating the inflammatory cytokines interleukin (IL)-18 (interleukin 18) and IL-1B (interleukin 1- beta) $)^{15,16}$. Increased expression of IL-18, ILIB, tumor necrosis factor-alpha, and interferon-gamma has been demonstrated in cases of Chagas cardiomyopathy in vivo ${ }^{17,18}$. The widespread involvement of myocardial cells in this inflammatory process results in scarring and fibrosis that contribute to the subsequent cardiac pathology ${ }^{12}$. These findings suggest that caspase-1 may play an important role in the development of $T$. cruzi-induced myocardial dysfunction.

Although polymorphisms of inflammatory cytokines (IL-18, CCR2 (C-C chemokine receptor type 2), CCR5 (C-C chemokine receptor type 5), LTA (lymphotoxin alpha), and TGF- $\beta$ (tumor growth factor-beta) $)^{19-24}$ have shown associations with an elevated risk of Chagas cardiomyopathy, polymorphisms of the caspase-1 gene (CASPl) have not previously been evaluated in patients with Chagas disease. Given that caspase-1 plays a pivotal role in the inflammatory cascade provoked by $T$. cruzi infection, we hypothesized that polymorphisms that affect caspase-1 expression could function as early indicators of Chagas cardiomyopathy risk. Accordingly, we evaluated the associations of two known CASP1 single nucleotide polymorphisms (SNPs) with the presence of Chagas cardiomyopathy in a Bolivian population.

\section{METHODS}

\section{Ethical considerations}

The study protocol was approved by the Institutional Review Board of Universidad Catolica Boliviana (Santa Cruz, Bolivia), PRISMA (Lima, Peru), Johns Hopkins Bloomberg School of Public Health (Baltimore, MD, USA), and University of California San Francisco School of Medicine (San Francisco, CA, USA). All participants provided written informed consent.

\section{Patient population and recruitment}

Recruitment occurred in the internal medicine ward, outpatient clinic, and waiting area of San Juan de Dios Hospital, the largest public general hospital in Santa Cruz, Bolivia. All adults 18 years or older were eligible to participate. Some participants, who were aware of the study, also came to the hospital for voluntary recruitment. Exclusion criteria included active hospitalization for a severe non-cardiac disease such as sepsis, chronic obstructive pulmonary disease, or chronic renal insufficiency, and pregnancy.

\section{Specimen handling and determination of Trypanosoma cruzi infection}

Blood samples were collected from patients, and genomic DNA (deoxyribonucleic acid) was extracted using the Roche High Pure PCR (polymerase chain reaction) Template Preparation Kit (Roche Diagnostics) according to the manufacturer instructions and stored at $-20^{\circ} \mathrm{C}$. Serum specimens were tested for T. cruzi infection by enzyme-linked immunoassay (Wiener Recombinante 3.0 ELISA (enzyme linked immunosorbent assay), Rosario, Argentina) and an indirect hemagglutination test (Chagas Polychaco kit, Lemos Laboratories, Santiago del Estero, Argentina). For specimens with discordant results, the trypomastigote excreted-secreted antigens blot assay was performed according to a previously published protocol ${ }^{25}$. T. cruzi infection was confirmed based on positive results by at least two of the above tests.

\section{Classification of cardiac status}

Participants were categorized according to T. cruzi infection status and cardiac severity class. Epidemiologic data were collected using a structured questionnaire. Cardiac severity was determined by electrocardiography (Welch-Allyn portable machine) and echocardiography (Sonosite Micromaxx ultrasound) evaluated by cardiologists blinded to patient infection status, and readings were provided in a structured data format. Class I was defined based on normal results on electrocardiogram and a normal ejection fraction $[(\mathrm{EF}) ;>50 \%]$ on echocardiogram ${ }^{26}$. Class II was defined if any of the following were present on echocardiography: intraventricular conduction delay, right bundle branch block, left bundle branch block, left anterior hemiblock, left posterior hemiblock, $1^{\text {st }}, 2^{\text {nd }}$ or $3^{\text {rd }}$ degree atrioventricular block, multifocal or paired ventricular premature beats, atrial fibrillation or flutter, pacemaker, atrioventricular dissociation or severe bradycardia $(<50 \mathrm{bpm})^{2,27}$. Participants were assigned to class III (EF 40-50\%) or to class IV $(\mathrm{EF}<40 \%)$ based on echocardiogram findings ${ }^{26}$. We followed the severity classification system published by Rassi et al. ${ }^{28}$. EF cut-offs for Class III and IV were based on the recommendations of the American Society of Echocardiography (ASE), in which an EF of $40-50 \%$ indicates mild to moderate global ventricular dysfunction (Class III) and an EF $<40 \%$ indicates severely abnormal function (Class IV) ${ }^{29}$.

\section{Single nucleotide polymorphisms selection and genotyping}

We selected two CASP1 SNPs for analysis: rs501192 (C_27136097_10) and rs570685 (C_ 962600_10). CASP1 rs501192 was selected because previous studies have shown an association of this SNP with non-Chagas heart disease ${ }^{30}$, and CASP1 rs570685 is a tag-SNP defining a CASP1 haplotype that is differentiated within Latin American populations. Data from the 1000 Genomes Project (http://www.1000genomes.org/) were used to compile $C A S P 1$ haplotypes, and linkage disequilibrium 
was assessed by Haploview (https://www.broadinstitute. org/scientific-community/science/programs/medical-andpopulation-genetics/haploview/haploview). Genotyping was performed using the Taqman SNP Genotyping Assay according to manufacturer's protocol (Applied Biosystems, Foster City, CA, USA). Non-DNA template controls and positive controls (Coriell DNA Laboratories, Camden, NJ) were genotyped concurrently also using manufacturer's protocol to ensure highquality genotyping ( $>95 \%$ call rate).

\section{Statistical analysis}

The target sample size for this study was determined to be $\mathrm{n}=66$ for all cases (cardiomyopathy Class II, III, IV, either $T$. cruzi positive or negative) and controls (cardiomyopathy Class I, either $T$. cruzi positive or negative) to be able to detect a difference of $20 \%$ (30\% for cases and $10 \%$ for controls) in SNP prevalence at a power of $80 \%$ with a $95 \%$ confidence interval.

For baseline characteristics, differences among continuous variables were evaluated by the Kruskal-Wallis test. Comparisons of dichotomous variables were analyzed using chi-squared or Fisher's exact tests, as appropriate. All groups were tested for markers of Hardy-Weinberg equilibrium by Fisher's exact test. Genotype frequencies were analyzed by three inheritance patterns (dominant, recessive, additive) using logistic regression adjusted for sex and age in the SNPassoc R package. Goodness of fit was assessed for each model by Akaike information criterion values. Statistical significance was defined as $p<0.05$.

\section{RESULTS}

Genotyping data were generated for 149 T. cruzi-infected $\left(T c^{+}\right)$and 87 uninfected $\left(T c^{-}\right)$individuals (Table 1). Among the $T C^{+}$participants, increasing severity of cardiac disease was associated with a progressively increasing median age $(\mathrm{p}=0.0019)$. This trend was also detected among uninfected participants, but it did not reach statistical significance. A higher proportion of $T c+$ Class I participants were female as compared to those in Class II $(\mathrm{p}=0.0327)$ or all patients in the $T c+$ cardiomyopathy group $(\mathrm{p}=0.0264)$. The sero-negative study population showed no significant differences in sex distribution according to cardiac class.

Among the $T c+$ participants, the AA allele for the CASP 1 SNP rs501192 was more prevalent in those with cardiomyopathy (Class II, III, and IV) compared to those with a normal cardiac status (Class I) (Table 2). This pattern was stronger when restricting this analysis to only the $T c+$ individuals of Class I and II, and approached statistical significance (OR -2.64 , $\mathrm{p}=0.064$ ). Among the uninfected participants, there was also a positive trend observed for carriers of these SNPs who had cardiomyopathy (Class II, III, and IV) compared to those of Class I (Table 2). However, neither SNP was associated with an altered risk of $T$. cruzi infection in this study population (Table 3).

\section{DISCUSSION}

To our knowledge, this is the first study to evaluate $C A S P 1$ polymorphisms in the context of Chagas cardiomyopathy.
Within our Bolivian cohort, we observed a stronger trend of an association of these polymorphisms with diseases in the Class I vs. Class II comparison for the $T c+$ group than when considering all of the $T c+$ cardiomyopathy subjects (Class II, III, IV) vs. those of Class I, despite the smaller sample size. This finding may reflect a higher specificity of the criteria for diagnosing class II for Chagas cardiomyopathy than those for classes III and IV, which require a decreased EF and thus may include patients with other etiologies of congestive heart failure. However, the positive trend for an association of both polymorphisms with cardiomyopathy among uninfected participants suggests that several caspase-1-related genes may be involved in cardiomyopathy development, in which inflammation is integral to the pathogenesis, regardless of the specific etiology.

Caspase-1 is the primary activator of the inflammatory cytokines IL-1B and IL-18, and plays a major role in the pathogenesis of viral myocarditis ${ }^{16,31,32}$. A previous study showed that heart tissue from patients with viral myocarditis had increased caspase-1 expression, and the intensity of expression was directly related to the severity of heart failure and lack of functional recovery at 6 months ${ }^{33}$. In vivo models of dilated cardiomyopathy also showed increased cleavage and activation of pro-caspase-1, resulting in increased myocardial inflammation and systolic dysfunction ${ }^{34}$. These studies reflect the acute inflammatory response to infection that can translate into the consequences observed with long-standing disease. Although caspase-1 polymorphisms have not previously been studied in Chagas disease, they have been linked to variations in IL-1B and IL-18 activity ${ }^{30}$. Moreover, polymorphisms of the cytokine IL-18 are known to be associated with susceptibility to Chagas cardiomyopathy ${ }^{19}$, providing a likely mechanism for our detected trends.

We chose to focus on the SNP rs501192 because of its known occurrence in Latin American populations based on the 1000 Genomes database. Paradoxically, this SNP was previously reported to be associated with a lower risk of coronary artery disease in a European study ${ }^{30}$. Therefore, further investigation will be required to understand this apparent inconsistency in two genetically different populations.

Many of the findings associated with early Chagas cardiomyopathy, including ST-T changes, abnormal Q waves on electrocardiogram, and microvascular abnormalities, have been attributed to an ongoing inflammatory process ${ }^{2}$. These indications are also the earliest signs of cardiomyopathy resulting from fibrosis, as seen by an increased myocardial signal on magnetic resonance imaging ${ }^{35}$. The SNPs rs501192 and rs570685 are both located in the intronic regions of CASPI (intron 6 and 2 , respectively), and could therefore play a role in splicing errors during transcription. Dysregulation of the inflammasome NLPR 3 due to genetic mutations of its related genes has been implicated in other inflammatory diseases such as cryopyrinassociated periodic syndromes, familial Mediterranean fever, pyogenic arthritis, pyoderma gangrenosum, and acne syndrome $^{36}$. Both the NLPR3 inflammasome and caspase-1 are also key players in the molecular mechanisms underlying 
TABLE 1

Median age, sex distribution, and genotype distribution for the $C A S P 1$ polymorphisms in $T c+$ and $T c$ - patients.

\begin{tabular}{|c|c|c|c|c|c|c|}
\hline \multirow[t]{5}{*}{ SNP } & \multirow[t]{5}{*}{ Allele } & \multirow[t]{5}{*}{ Total (N) } & \multicolumn{4}{|c|}{ Serology } \\
\hline & & & \multicolumn{4}{|c|}{$T c+$} \\
\hline & & & \multicolumn{4}{|c|}{ Cardiac classification } \\
\hline & & & $\mathbf{I}$ & II & III & IV \\
\hline & & & N (\%) & N (\%) & $\mathbf{N}(\%)$ & N (\%) \\
\hline \multirow[t]{3}{*}{ Rs501192 } & GG & 101 & $31(41.8)$ & $22(42.3)$ & $5(35.7)$ & $7(77.7)$ \\
\hline & GA & 98 & $36(48.6)$ & $19(36.5)$ & $6(42.8)$ & $2(22.2)$ \\
\hline & $\mathrm{AA}$ & 37 & $7(9.4)$ & $11(21.1)$ & $3(21.4)$ & $0(0.0)$ \\
\hline \multirow[t]{3}{*}{ Rs570685 } & AA & 72 & $24(32.4)$ & $18(34.6)$ & $4(28.5)$ & $4(44.4)$ \\
\hline & $\mathrm{AC}$ & 109 & $37(50.0)$ & $19(36.5)$ & $6(42.8)$ & $4(44.4)$ \\
\hline & $\mathrm{CC}$ & 55 & $13(7.5)$ & $15(28.8)$ & $4(28.5)$ & $1(11.1)$ \\
\hline Sex [female] & & & $64.0 \%$ & $44.0 \%$ & $43.0 \%$ & $56.0 \%$ \\
\hline \multirow[t]{6}{*}{ Median Age [IQR] } & & & 60 & 55.5 & 64 & 65 \\
\hline & & & [60-65] & [42.2-65.7] & [55-68.5] & [55.5-68.5] \\
\hline & & & \multicolumn{4}{|c|}{$T c^{-}$} \\
\hline & & & \multicolumn{4}{|c|}{ Cardiac classification } \\
\hline & & & I & II & III & IV \\
\hline & & & N (\%) & N (\%) & N (\%) & N (\%) \\
\hline \multirow[t]{3}{*}{ Rs501192 } & GG & 101 & $24(45.2)$ & $4(21.0)$ & $2(40.0)$ & $6(60.0)$ \\
\hline & GA & 98 & $22(41.5)$ & $9(47.3)$ & $2(40.0)$ & $2(20.0)$ \\
\hline & AA & 37 & $7(13.2)$ & $6(31.5)$ & $1(20.0)$ & $2(20.0)$ \\
\hline \multirow[t]{3}{*}{ Rs570685 } & AA & 72 & $15(28.3)$ & $3(15.7)$ & $2(40.0)$ & $2(20.0)$ \\
\hline & $\mathrm{AC}$ & 109 & $28(52.8)$ & $8(42.1)$ & $2(40.0)$ & $5(50.0)$ \\
\hline & $\mathrm{CC}$ & 55 & $10(18.8)$ & $8(42.1)$ & $1(20.0)$ & $3(30.0)$ \\
\hline Sex [female] & & & $66.0 \%$ & $58.0 \%$ & $60.0 \%$ & $40.0 \%$ \\
\hline \multirow{2}{*}{$\begin{array}{l}\text { Median age } \\
\text { (interquartile range) }\end{array}$} & & & 60 & 52 & 54 & 60 \\
\hline & & & $(60-62)$ & $(45-65)$ & $(44-70)$ & $(48-67)$ \\
\hline
\end{tabular}

CASP1: caspase-1; Tc+: Trypanosoma cruzi positive; Tc: Trypanosoma cruzi negative; SNP: single nucleotide polymorphism; IQR: interquartile rang.

other diseases in which inflammation plays a critical role in the pathophysiology, such as psoriasis, gout, and Alzheimer's disease $^{36,37}$. Our data suggest that the genetic influences of NLPR3 inflammasome and caspase-1 merit further investigation in mediating the pathology and risk of Chagas disease as well.

Our study was limited by its relatively small sample size and cross-sectional design, and the relative infrequency of patients with advanced cardiomyopathy in the study population. These factors limited our statistical power for the case-control analysis. Therefore, examination of both CASP1 polymorphisms in a larger study population will be needed to provide more robust results. Ideally, future studies would be longitudinal in design to provide unambiguous data on the progression of Chagas cardiomyopathy. We attempted to recruit case and control groups with similar age and sex distributions; however, this was not easily achieved because the natural history of cardiomyopathy inevitably leads to older age in groups with more severe disease. In addition, women were more likely to be available and/or willing to participate than men. Lastly, we did not obtain consent to generate genetic data related to ethnicity, and therefore, 
TABLE 2

Association of CASP1 polymorphisms between subjects with Class I and Class II/III/IV cardiomyopathy according to infection status, adjusted for age and sex.

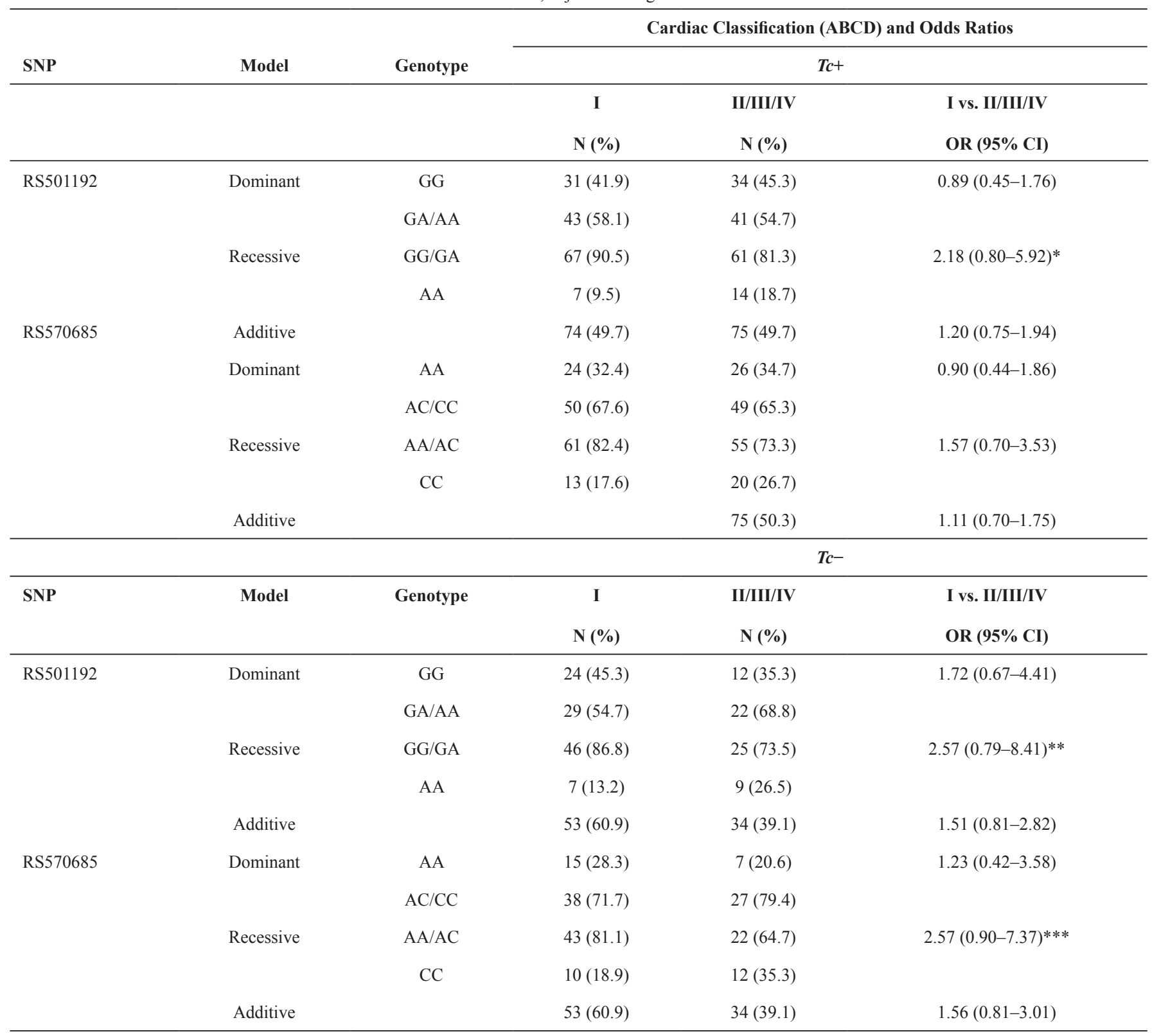

CASP1: caspase 1; ABCD: Cardiac classification; SNP: single nucleotide polymorphism; OR: odds ratios. ${ }^{*} \mathrm{p}=0.117 .{ }^{* *} \mathrm{p}=0.115 .{ }^{* * *} \mathrm{p}=0.075$.

the potential influence of genetic background could not be evaluated. However, the majority of patients at this hospital are known to be of mixed indigenous and European descent.

Our results suggest an etiologic role of CASP1 genetic variations in the risk of Chagas cardiomyopathy, which shows potential for development as a genetic biomarker to identify asymptomatic $T c^{+}$patients at high risk of subsequent cardiomyopathy. The current available prognostic markers are only able to indicate late-stage findings (i.e., New York Heart Association Class III/IV, cardiomegaly, impaired left ventricular systolic function, and ventricular arrhythmias on ambulatory monitoring) that occur long after anti-parasitic treatment can have any impact ${ }^{6,9}$. Therefore, infected individuals who carry the SNP of interest could be flagged as high priority for antitrypanosomal treatment.

In summary, the results of the present study suggest that caspase-1 variants may contribute to Chagas cardiomyopathy development, highlighting the need for continued research into caspase- 1 as a mediator of inflammation, preferably in robust, longitudinal study designs. 
TABLE 3

Association of CASP1 polymorphisms between asymptomatic (I) and cardiomyopathy patients (II/III/IV) according to infection status, adjusted for age and sex.

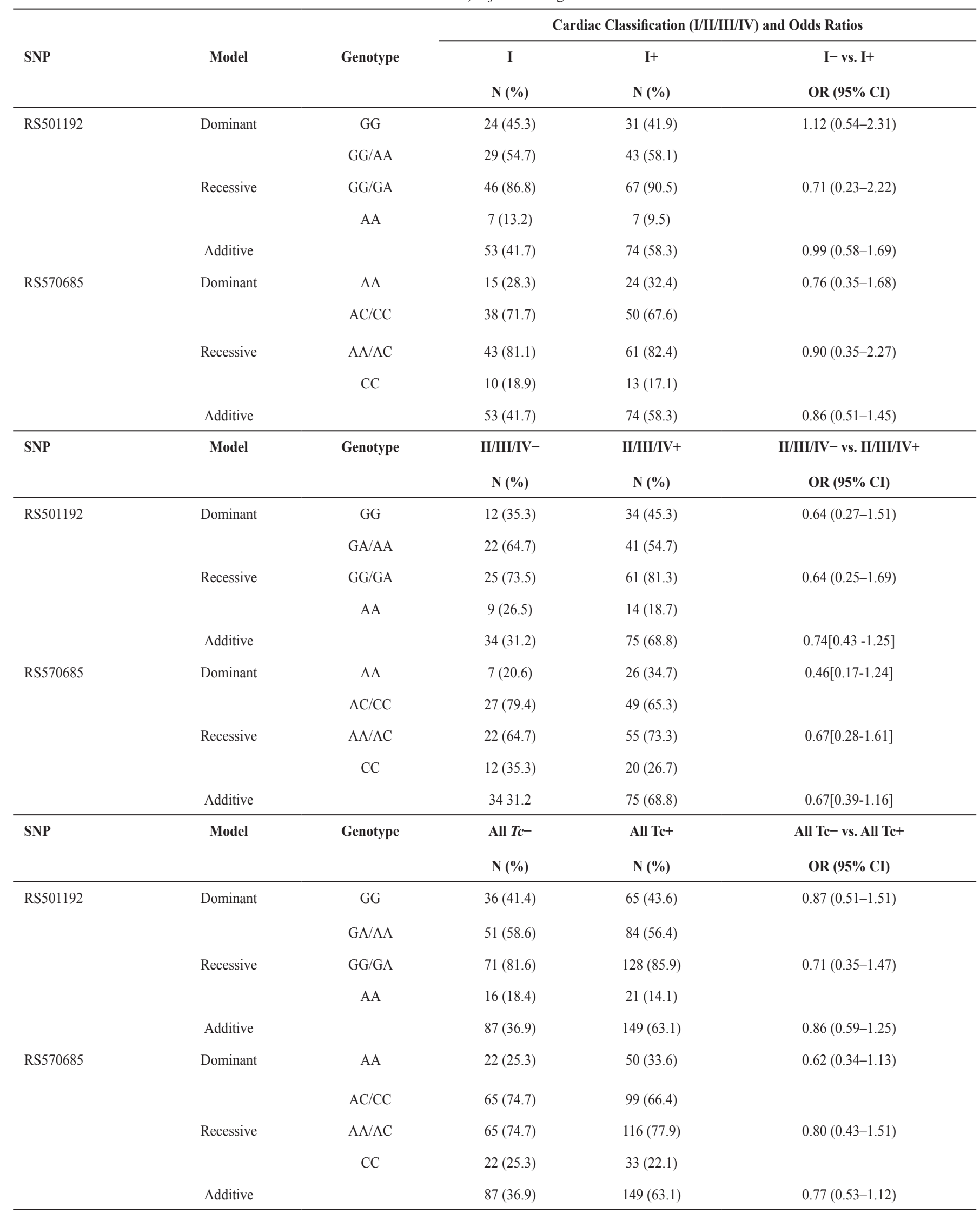

CASP1: caspase 1; SNP: single nucleotide polymorphism; OR: odds ratios; Tc+: Trypanosoma cruzi positive; Tc-: Trypanosoma cruzi negative. 


\section{Acknowledgements}

We thank Roxana Bravo Nevarro, Margot Ramirez Jaldin, Eliana Saenza Vasquez, Antonio Fernandez, Rose Do, Manuela Verastegui, Yomara Lazaro, Lidabel Rios, Patricia Sheen, Monica Pajuelo, and Cristina Maset Viader for their contributions to this work.

\section{Conflict of interest}

The authors declare that there is no conflict of interest.

\section{Financial support}

This work was supported by NIH R01-AI107028-01A1 and in part by the Doris Duke Charitable Foundation through a grant supporting the Doris Duke International Clinical Research Fellows Program at the University of California San Francisco. Katherine Fu is a Doris Duke International Clinical Research Fellow. The funding sources had no role in the study design, collection, analysis and interpretation of the data, preparation of the manuscript, or the decision to submit the manuscript for publication.

\section{REFERENCES}

1. World Health Organization. Global Burden of Disease Estimates for 2000-2012. Geneva: World Health Organization; 2014. Available at: http://www.who.int/healthinfo/global_burden_disease/estimates/ en/index 2.html.

2. Bern C, Montgomery SP, Herwaldt BL, Raasi A, Marin-Neto JA, Dantas RO, et al. R Evaluation and treatment of Chagas disease in the United States. A systematic review. JAMA Clin Rev. 2007;298(18):2171-81.

3. Raasi Jr A, Rassi A, Marcondes de Rezende J. American trypanosomiasis (Chagas disease). Infect Dis Clin North Am. 2012;26(2):275-91.

4. Rassi Jr A, Rassi A, Marin-Neto JA. Chagas disease. Lancet. 2010;375(9723):1388-1402.

5. Hidron AI, Gilman RH, Justiniano J, Blackstock AJ, Lafuente C, Selum W, et al. Chagas cardiomyopathy in the context of the chronic disease transition. PLoS Negl Trop Dis. 2010;4(5):e688.

6. Rassi Jr A, Rassi A, Little WC, Xavier SS, Raasi SG, Rassi AG, et al. Development and Validation of a risk score for predicting death in Chagas' heart disease. N Engl J Med. 2006;355(8):799-808.

7. de Andrade AL, Zicker F, de Oliveira RM, Almeida Silva S, Luquetti A, Travassos LE, et al. Randomised trial of efficacy of benznidazole in treatment of early Trypanosoma cruzi infection. Lancet (London, England). 1996;348(9039):1407-13.

8. Sosa-Estani S, Segura EL, Ruiz AM, Velazquez E, Porcel BM, Yampotis C. Efficacy of chemotherapy with benznidazole in children in the indeterminate phase of Chagas' disease. Am J Trop Med Hyg. 1998;59(4):526-9.

9. Morillo CA, Marin-Neto JA, Avezum A, Sosa-Estani S, Rassi Jr A, Rosas F, et al. Randomized trial of benznidazole for chronic Chagas' cardiomyopathy. N Engl J Med. 2015;373(14):1295306.

10. Marin-Neto JA, Cunha-Neto E, Maciel BC, Simões MV. Pathogenesis of chronic Chagas heart disease. Circulation. 2007;115(9):1109-23.

11. Machado FS, Dutra WO, Esper L, Gollon KJ, Teixeira MM, Factor $\mathrm{SM}$, et al. Current understanding of immunity to Trypanosoma cruzi infection and pathogenesis of Chagas disease. Semin Immunopathol. 2012;34(6):753-70.
12. Bern C. Antitrypanosomal therapy for chronic Chagas' disease. N Engl J Med. 2011;364(26):2527-34.

13. Silva GK, Costa RS, Silveira TN, Caetano BC, Horta CV, Gutierrez FR, et al. Apoptosis-associated speck-like protein containing a caspase recruitment domain inflammasomes mediate IL-1 $\beta$ response and host resistance to Trypanosoma cruzi infection. J Immunol. 2013;191(6):3373-83.

14. Latz E, Xiao TS, Stutz A. Activation and regulation of the inflammasomes. Nat Rev Immunol. 2013;13(6):397-411.

15. Gonçalves VM, Matteucci KC, Buzzo CL, Miollo BH, Ferrante D, Torrecilhas AC, et al. NLRP3 controls Trypanosoma cruzi infection through a caspase-1-dependent IL-1R-independent NO production. PLoS Negl Trop Dis. 2013;7(10):e2469.

16. Gu Y, Kuida K, Tsutsui H, Ku G, Hsiao K, Fleming MA, et al. Activation of interferon-gamma inducing factor mediated by interleukin-1beta converting enzyme. Science. 1997;275(5297): 206-9.

17. Gomes JAS, Bahia-Oliveira LMG, Rocha MOC, Martins-Filho OA, Gazzinelli G, Correa-Oliveira R. Evidence that development of severe cardiomyopathy in human Chagas' disease is due to a Th1specific immune response. Infect Immun. 2003;71(3):1185-93.

18. Abel LC, Rizzo LV, Ianni B, Albuquerque F, Bacal F, Carrara D, et al. Chronic Chagas' disease cardiomyopathy patients display an increased IFN $-\gamma$ response to Trypanosoma cruzi infection. J Autoimmun. 2001;17(1):99-107.

19. Nogueira LG, Frade AF, Ianni BM, Laugier L, Pissetti CW, Cabantous S, et al. Functional IL18 polymorphism and susceptibility to chronic Chagas dsease. Cytokine. 2015;73(1):79-83.

20. Machuca MA, Suárez EU, Echeverría LE, Martín J, González CI. SNP/haplotype associations of CCR2 and CCR 5 genes with severity of chagasic cardiomyopathy. Hum Immunol. 2014;75(12):1210-5.

21. Pissetti CW, Oliveira RF de, Correia D, Nascentes GAN, Llaguno MM, Rodrigues Jr V. Association Between the Lymphotoxin-Alpha Gene Polymorphism and Chagasic Cardiopathy. J Interf Cytokine Res. 2013;33(3):130-5.

22. Calzada JE, Beraún Y, González CI, Martín J. Transforming growth factor beta 1 (TGF $\beta 1$ ) gene polymorphisms and Chagas disease susceptibility in Peruvian and Colombian patients. Cytokine. 2009;45(3):149-53.

23. Vasconcelos RHT, Montenegro SML, Azevedo EAN, Gomes YM, Morais CNL. Genetic susceptibility to chronic Chagas disease: an overview of single nucleotide polymorphisms of cytokine genes. Cytokine. 2012;59(2):203-8.

24. Flórez O, Martín J, González CI. Genetic variants in the chemokines and chemokine receptors in Chagas disease. Hum Immunol. 2012;73(8):852-8.

25. Umezawa ES, Nascimento MS, Kesper Jr N, Coura JR, BorgesPereira, Junqueira AC, et al. Immunoblot assay using excretedsecreted antigens of Trypanosoma cruzi in serodiagnosis of congenital, acute, and chronic Chagas' disease. J Clin Microbiol. 1996;34(9):2143-7.

26. Hunt SA, Abraham WT, Chin MH, Feldman AM, Francis GS, Ganiats TG, et al. Guideline Update for the Diagnosis and Management of Chronic Heart Failure in the Adult: A Report of the American College of Cardiology/American Heart Association Task Force on Practice Guidelines (Writing Committee to Update the 2001 Guidelines for the Evaluation and Management of Heart Failure). Circulation. 2005;112(12):e154-e235.

27. Okamoto EE, Sherbuk JE, Clark EH, Marks MA, Gandarilla O, Galdos-Cardenas G, et al. Biomarkers in Trypanosoma cruziinfected and uninfected individuals with varying severity of 
cardiomyopathy in Santa Cruz, Bolivia. PLoS Negl Trop Dis. 2014;8(10):e-3227.

28. Rassi Jr A, Dias JCP, Marin-Neto JA, Rassi A. Challenges and opportunities for primary, secondary, and tertiary prevention of Chagas' disease. Heart. 2009;95(7):524-34.

29. Lang RM, Bierig M, Devereux RB, Flachskampf FA, Foster E, Pllikka PA, et al. Recommendations for chamber quantification: a report from the American Society of Echocardiography's Guidelines and Standards Committee and the Chamber Quantification Writing Group, developed in conjunction with the European Association of Echocardiograph, a branch of the European Society of Cardiology. J Am Soc Echocardiogr. 2005;18(12):1440-63.

30. Blankenberg S, Godefroy T, Poirier O, Rupprecht HJ, Barbaux S, Bickel C, et al. Haplotypes of the caspase-1 gene, plasma caspase-1 levels, and cardiovascular risk. Circ Res. 2006;99(1):102-8.

31. Cerretti DP, Kozlosky CJ, Mosley B, Nelson N, Van Ness K, Greenstreet RA, et al. Molecular cloning of the interleukin-1 beta converting enzyme. Science. 1992;256(5053):97-100.
32. Wang Y, Gao B, Xiong S. Involvement of NLRP3 inflammasome in CVB3-induced viral myocarditis. Am J Physiol Heart Circ Physiol. 2014;307(10):H1438-47.

33. Toldo S, Kannan H, Bussani R, Anzini M, Sonnino C, Sinagra G, et al. Formation of the inflammasome in acute myocarditis. Int $\mathrm{J}$ Cardiol. 2014;171(3):119-21.

34. Bracey NA, Beck PL, Muruve DA, Hirota SA, Guo J, Jabagi H, et al. The Nlrp3 inflammasome promotes myocardial dysfunction in structural cardiomyopathy through interleukin-1 $\beta$. Exp Physiol. 2013;98(2):462-72.

35. Torreão JA, Ianni BM, Mady C, Naia E, Rassi CH, Nomura C, et al. Myocardial tissue characterization in Chagas' heart disease by cardiovascular magnetic resonance. J Cardiovasc Magn Reson. 2015;17(1):97.

36. Denes A, Lopez-Castejon G, Brough D. Caspase-1: is IL-1 just the tip of the ICEberg? Cell Death Dis. 2012;3(7):e338.

37. Freeman LC, Ting JP-Y. The pathogenic role of the inflammasome in neurodegenerative diseases. J Neurochem. 2016;136(Suppl 1):29-38. 